\title{
On Predicting Learning Styles in Conversational Intelligent Tutoring Systems using Fuzzy Classification Trees
}

\author{
Keeley Crockett, Annabel Latham, David Mclean, Zuhair Bandar, James O'Shea \\ The Intelligent Systems Group, School of Computing, Mathematics and Digital Technology, \\ The Manchester Metropolitan University, Chester Street, \\ Manchester, M1 5GD, UK \\ K.Crockett@mmu.ac.uk
}

\begin{abstract}
Oscar is a conversational intelligent tutoring system (CITS) which dynamically predicts and adapts to a student's learning style throughout the tutoring conversation. Oscar aims to mimic a human tutor to improve the effectiveness of the learning experience by leading a natural language tutorial and adapting material to suit an individual's learning style. Prediction of learning style is undertaken through capturing independent variables during the conversation. The variable with the highest value determines the individuals learning style. This paper proposes a new method which uses a fuzzy classification tree to build a fuzzy predictive model using these variables which are captured through natural language dialogue Experiments have been undertaken on two of the learning style dimensions: perception (sensory-intuitive) and understanding (sequential-global). Early results show the model has substantially increased the predictive accuracy of the Oscar CITS and discovered some interesting relationships amongst these variables.
\end{abstract}

Keywords- Fuzzy classification tree, conversational agent; intelligent tutoring systems;

\section{INTRODUCTION}

Intelligent Tutoring Systems (ITS) are computer-based educational systems which employ intelligent technologies to provide individualised instruction [1]. In order to personailise the students learning experience, a number of factors can be used such as user affect, such as emotion [2], personality [3] and learning style [4]. Learning styles recognise that people have instructional preferences in how they absorb and process information. One of the most established learning style models is known as the Index of Learning Styles model (ILSM). ILSM was proposed by Felder and Silverman [5] to describe the learning styles in engineering education and suggested different teaching styles to address learners' needs. Each person has their own learning strategy for learning specific subject matter in a particular context [5]. If the teacher knows the students' learning styles then delivery of a subject can be adapted.

Existing ITS capture learning styles using a formal questionnaire [6], [7] or by analysing a student's behaviour within the ITS [8], [9]. The main problem with questionnaires is that they are time consuming and onerous and people do not tend to complete them accurately. Predicting learning style using a history of student behaviour means adaptation is delayed until several modules have been completed, and also a student's learning style may change over time or for different topics. EDUCE [10] and WELSA [11] both estimate learning style dynamically for curriculum sequencing, but do not include a conversational interface or incorporate other intelligent tutoring technologies.

Most ITS do not have a natural language interface but rely instead on navigation around the tutoring material through buttons and hyperlinks. This is due to the fact that conversational interfaces are complex to develop [6..11]. However, to adequately mimic a human tutor, an ITS should support the construction of knowledge through discussion [12]. One way of providing a natural language interface is through the incorporation of a Conversational Agent (CA) [13]. A CA uses natural language dialogue to communicate with users [14] and will have the ability to reason and pursue a course of action based on its interactions with humans and other agents. Some work has been done in incorporating CAs with ITS but this has been to help the learning management system, rather than undertake a tutorial with a learner $[15,16]$. AutoTutor [16] is a conversational intelligent tutoring system (CITS), which helps students construct knowledge about computer literacy and physics. CIRCSIM-tutor [16] engages students in discussion to solve physiology problems. Discussions that take place in either system do not consider learning styles during tutoring. Knowledge of an individual's learning style is essential when developing a CITS which can adapt its teaching style to individual learner preferences [12].

Oscar is a CITS that dynamically predicts learning style throughout the tutoring conversation and adapt its intelligent tutoring style to suit an individual's learning style [12]. Prediction of learning style occurs during the conversational tutoring session by capturing the values independent variables. The variable with the highest value determines the individuals learning style in accordance with the Index of Learning Styles Model (ILSM) [5]. The current method has had some degrees of success but its fundamental weakness is that it does not consider the relationships between these variables in predicting the learning style.

Fuzzy models have been developed for educational learning since 1996 when Hawkes and Derry [18] proposed 
a model that captured the way in which human tutor's evaluated borderline tutoring decisions in accordance with student behavior. One of the most recent areas of research is in making learning object recommendations for a specific domain based on an individual's learning style [19..22]. In [20] a fuzzy inference system comprising of six rules is applied to a recommender system in order to assign a student a suitable learning object in the field of computer networks. Cabada et al [21] used a combined fuzzy-neuro approach to recommend learning objects. The approach learns student behavior based upon types of tutoring materials students have previously used and their learning styles. In [22] a fuzzy-neuro approach was used to infer the learning characteristics of a student in an interactive learning environment. This method was used to obtain an optimal learning path both instructor and learner through the tutoring domain. Whilst each of the above systems have shown degrees of success using small experimental groups none attempt to predict the learning style through obtaining variables captured through natural language dialogue which occurs naturally through a tutoring conversation.

This paper proposes an approach to modeling these variables using fuzzy classification trees in order to improve the predictive accuracy. The concepts of fuzzy decision trees are well established [23..27]. The main features of fuzzy trees, compared with crisp trees, are the gradual transitions that exist between continuous attribute values and the inference process that allows all branches within the tree to be fired to some degree. Existing methodologies for creating fuzzy trees present different approaches on how to determine fuzzy regions at nodes within the tree [28] and the fuzzy inference strategy which is applied [23-24]. In this paper, a modified version of the Fuzzy Inference Algorithm (FIA) [24] is used to produce a fuzzy predictive model using variables extracted through natural language dialogue during the tutoring conversation.

The paper is organized as follows: Section II describes Oscar, the conversational intelligent tutoring system used within this paper. Section III describes the modified FIA and how it has been applied to the learner's data set. Section IV explains the experimental process which was undertaken on two of the learners dimensions: perception (sensoryintuitive) and understanding (sequential-global) [5]. Finally section $\mathrm{V}$ presents the results and discussion.

\section{OSCAR: A CONVERSATIONAL INTELLIGENT TUTORING SYSTEM}

The Oscar CITS is a conversational intelligent tutoring system designed to dynamically predict a student's learning style during a tutoring conversation, and to adapt the tutoring style to suit the individual learner [17]. Oscar's pedagogical aim is to provide the learner with the most appropriate learning material for their learning style leading to a more effective learning experience and a deeper understanding of the topic. Rather than being designed with the purpose of picking up learning styles (such as [5]), the Oscar CITS attempts to mimic a human tutor by leading a two-way discussion and using cues from the student dialogue and behaviour to predict and adapt to their learning style. Oscar's natural dialogue interface and classroom tutorial style are intuitive to learners, enabling them to draw on experience to feel more comfortable and confident in using the CITS. Oscar CITS is a personalised tutor which can answer questions, provide hints and assistance using natural dialogue, and which favours learning material to suit each individual's learning style. The Oscar CITS offers 24hour personalised learning support at a fixed cost. Oscar's intelligent approach includes presenting learning material in the sequence and style most suited to the individual's learning style (curriculum sequencing [15]), analysing and giving feedback on incomplete and erroneous solutions (intelligent solution analysis [29]) and giving intelligent hints and discussing questions (problem solving support [30]). By combining all three intelligent technologies with a conversational interface, Oscar's intelligent support aims to build the confidence of the learner and improve motivation and deep understanding of the subject.

\section{A. The Index of Learning Styles}

In their Index of Learning Styles (ILS) model [5], Felder and Silverman described the learning styles in engineering education and suggested different teaching styles to address learners' needs. The ILS model defines four separate dimensions of preferred learning style, each relating to a step in the process of receiving and processing information as follows:

- Perception - this dimension categorises learners as sensory (SNS) or intuitive (INT) and relates to the type of information a student prefers to perceive (e.g. external, by observation (sensory) or internal, by speculation (intuitive)).

- Input - this dimension classes learners as visual or verbal by considering the way in which learners prefer to receive external information (e.g. by looking at diagrams (visual) or by listening to explanations (verbal)).

- Processing - this dimension groups learners as active or reflective according to the way perceived information is converted into knowledge (e.g. by the active discussion (active) or introspective consideration (reflective) of a topic).

- Understanding - this dimension categorises learners as sequential (SEQ) or global (GLO) depending on the way they progress towards understanding (e.g. by continual steps (sequential) or holistically in large jumps (global)).

Learning styles are central to the decision making within Oscar CITS. Following an initial study, a subset of the best ILS predictor questions for each learning style dimension was identified [31] and this was used to generate tutoring material for each learning style within Oscar.

For the purpose of this work, a conversational tutorial on structured query language (SQL) was developed. The target audience for the pilot study would be undergraduate computing students, for whom a Databases course including SQL is compulsory. Tutoring revision scenarios were designed based around the syllabus and the database 
lecturers' experience of revision tutorials. Each revision question was mapped to the ILS model using the model's descriptions of indicative behaviour, such as a preference for theoretical questions.

\section{B. Prediction and Adaptation}

The Oscar CITS aims to mimic a human tutor by using knowledge of learning styles and cues from student behaviour to predict individual learning style. During a tutorial, information is recorded about learner behaviours and then used to predict learning style. The experiments reported in $[12,31]$ compared the results of the formal ILS questionnaire to student behaviour to compute an accuracy of prediction based on 17 hypotheses. Three of the 17 hypotheses are shown as an example below.

H1: a student's learning path through the tutorial is indicative of learning style.

$\mathrm{H} 2$ : choosing to be guided through a process (or not) is indicative of learning style.

H3: The amount of discussion a student enters into with the tutor is indicative of learning style.

The variables with the best accuracy for an ILS dimension are then used to predict student learning style and adapt the tutoring style accordingly.

Oscar CITS uses an algorithm to determine the best adaptation for each tutoring question based on the student learning style strengths. The algorithm is fairly complex in that it considers all four learning style dimensions. Rather than simply adapting to the strongest student learning style, the algorithm separately considers each question and its ability to adapt for certain learning styles as well as a student's learning styles. This allows for tutorial questions where it is not possible to include adaptive learning material for all learning styles, as occur in real life. The development of the adaptive Oscar CITS is reported in [17].

Figure 1 shows two examples of logic rules used by the system to increment learning style values during tutoring. Learning styles are held in eight values within the student model, representing each pole of the four dimensions (perception (sensory-intuitive), input (visual-verbal), processing (active-reflective) and understanding (sequentialglobal) [17].
1. Example rule to test whether presenting information visually helps the student's information perception:

IF student does not know the answer

THEN show student image/diagram;

IF student shown image/diagram

AND student gives correct answer

THEN increase VISUAL;

2. Example rule to test how comfortable the student is with words and with detail:
IF answer is given in the explanation text
AND student does not know the answer
THEN increase INTUITOR
AND increase VISUAL;

Fig. 1. Example of logic rules used to adjust student learning style based on tutoring conversation

\section{Conversational Agent and Dialogue Design}

Oscar was developed using a type of conversational agent (CA) known as a goal-orientated conversational agent (GOCA) [13]. GO-CA's are a specific family of conversational agents that are designed to converse with humans through the use of natural language dialogue to achieve a specific task. In this case to act as an intelligent tutor to guide the learner through the tutorial based upon their learning style. The GO-CA used in this work utilised pattern matching algorithms to capture the values of specific attributes through dialogue interaction with a user. This is achieved through the use of scripts which contain sets of rules about the domain (SQL) and a knowledge base to guide the conversation towards achieving a specific goal (Oscar adaptation rules). In the Oscar CITS, the dialogue for each tutorial was scripted using Convagent's GO-CA [19]. Overall, there were 38 contexts containing around 400 rules. The full architecture and methodology for developing Oscar CITS is described in [32]. Throughout tutoring, the CITS records and logs information about the behaviour of the student, such as timing of interactions, the number of words used, the number of times FAQs are asked and the type of tutor resource accessed. The tutoring conversation is also recorded, along with information about the student knowledge of the topic being discussed and the adaptation employed.

Figure 2 shows a screenshot of the Oscar CITS user interface showing a user interaction where the student is being asked to write an SQL query with the results limited by two values. Oscar is guiding the student through each step of writing the query. The chat window shows the student's previous utterance, which is incorrect, and Oscar's response, followed by a reminder of the question. An image relevant to the stage of the query is shown to the right, and movies pop up in a new window if appropriate. Oscar's response helps the student by hinting at the solution by recommending an approach to writing the WHERE clause, rather than simply showing the answer. By adopting the constructivist style of teaching like human tutors, Oscar endeavors to provoke a deeper understanding of a topic. 


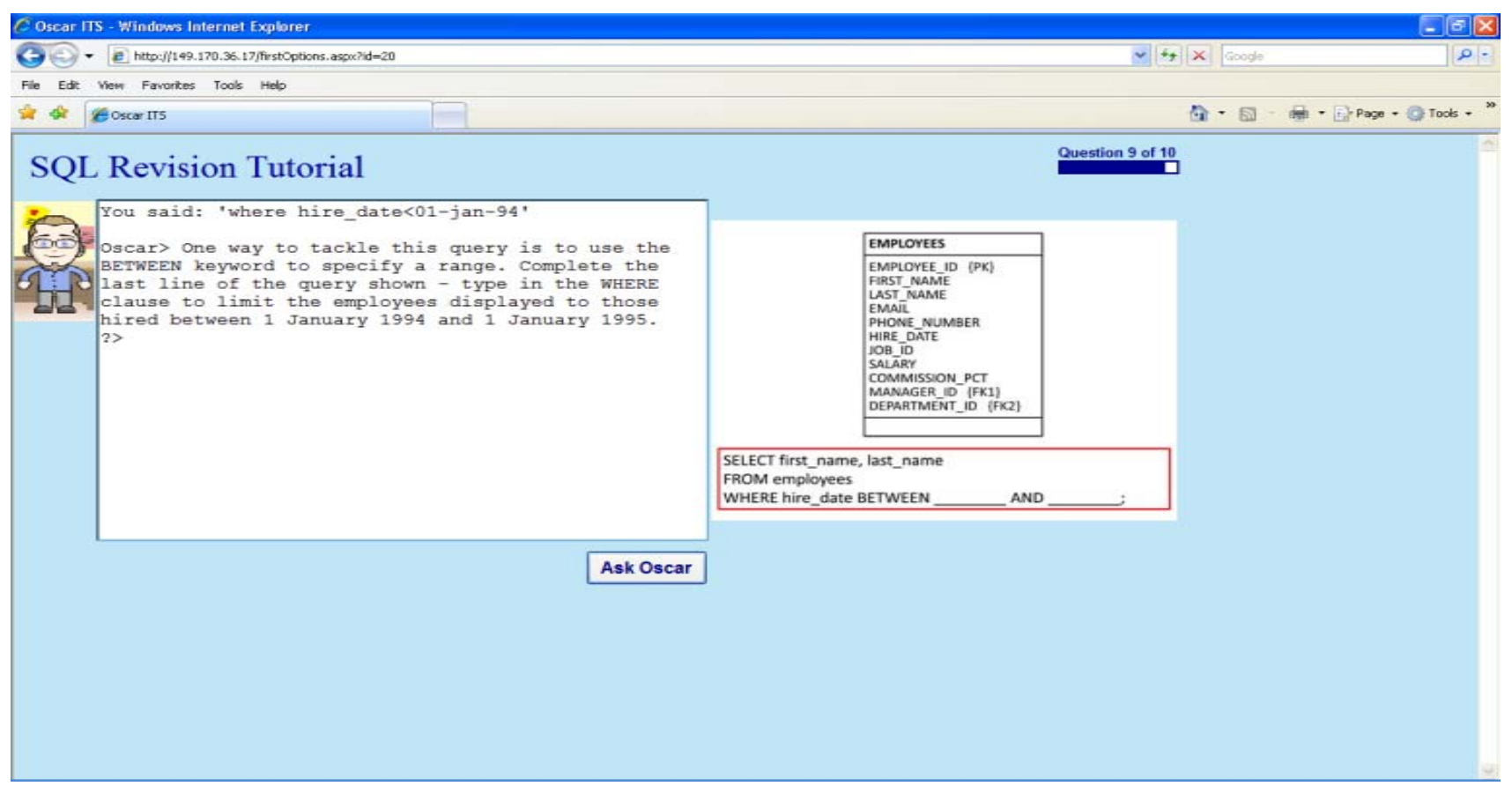

Fig. 2. Oscar CITS

\section{Example Learner Dialogue}

Table I shows part of a student's tutoring dialogue logged during experiments used for this work. In utterance 33, Oscar continues a question about data types. The student gives the wrong data type in utterance 34 , and next Oscar indicates that the answer is wrong and gives the student a second chance to answer in case of spelling errors, repeating the question. In utterance 36 the student tries again, still with the wrong answer. Oscar gives feedback that the answer is wrong (utterance 37), and helps by showing an image of the most common data types to see if the student is able to pick out the required answer. In this action, Oscar is helping the student learn by reminding them of the common data types and asking them to think about the correct data type rather than simply telling them the answer. In utterance 38 the student enters the correct data type, extended to include the decimal places, and Oscar then confirms that they have the right answer and repeats the solution to reinforce the knowledge.

TABLE I. EXAMPLE TUTORING DIALOGUE SNIPPET LOGGED DURING THE EXPERIMENT.

\begin{tabular}{l|l}
\hline Utterance & Dialogue \\
\hline \hline 33. Oscar> & $\begin{array}{l}\text { Now, in our books table suppose we want to store the price } \\
\text { of the book where the maximum price of a book is 99.99. } \\
\text { What would be the datatype? }\end{array}$ \\
VARCHAR2 \\
34. Student> \\
I'm sorry, that's wrong. Check your spelling and try again - \\
type in the datatype for book price (with a maximum of \\
99.99). \\
VARCHAR2 MAX 99.99 \\
That's still wrong. Take a look at the table of common \\
datatypes - can you now type in the datatype for book- \\
price where the maximum price is 99.99? \\
NUMBER(2,2) \\
37. Oscar $>$
\end{tabular}

\section{GENERATING FUZZY CLASSIFICATION TREES}

Previous work $[23,24,28]$ has shown that the generation of a fuzzy classifier from crisp induced trees produces a generalized and robust rule set without the need for human expert intervention during the fuzzification process. For the purpose of this paper the fuzzy classification trees will be constructed using the FIA algorithm [24] which will now be briefly described. FIA is an algorithm designed to build fuzzy classification trees from raw datasets. One of the strengths of the algorithm is that no pre-fuzzification of the data is required. FIA provides a mechanism for the direct fuzzification of both discrete and continuous attributes and the ability to use any T-norm/ T-conorm pair for determining fuzzy inference

\section{A. Fuzzy Inference Algorithm (FIA)}

A high level description of the stages of FIA is as follows:

1. Generate a crisp classification decision tree using predefined pruning criteria (in this work Quinlan's C4.5 decision tree algorithm was used [32]).

2. For each node, $i$ within the tree identify whether discrete or continuous attributes and define an opposing membership function pair. Each membership function will have an associated domain $\left(d m_{\mathrm{i}}, d n_{\mathrm{i}}\right)$ whose scope is determined by the attribute at that specific branch which is defined as

$$
d m_{i}=d t_{i}-n_{j} \sigma_{i} \text { and } \quad d n_{i}=d t_{i}+n_{j+l} \sigma_{i}
$$

Where $\sigma_{i}$ is the standard deviation of attribute $i$,

$n$ is a real number $\mathrm{n} \rightarrow[0,5]$ used to extend fuzziness 
across the domain of the attribute,

$d t$ is the decision threshold and

$d m$ and $d n$ are the lower and upper bounds respectively of membership function $i$.

3. Select T-norm pair with inference strategy.

Let a set of data $\boldsymbol{S}$ consist of $\boldsymbol{i}$-attributes $\left\{\boldsymbol{A}_{1}, \boldsymbol{A}_{2} \ldots \boldsymbol{A}_{\boldsymbol{i}}\right\}$ of domain D which are used to describe a single object. The process of learning from $\boldsymbol{S}$ involves a transformation function $\boldsymbol{F}$ which accepts as input $\boldsymbol{S}$ and produces a defuzzified outcome $\boldsymbol{O}$, which is a mapping

$$
F(S) \rightarrow O
$$

Applying an inference technique onto an existing tree consisting of $\boldsymbol{x}$ branches involves the combination of $\boldsymbol{V}$ membership function values $\left\{\mu_{1}, \mu_{2}, \ldots \mu_{v}\right\}$ of all root to leaf node paths. Let $\boldsymbol{T}$ be a set of all possible outcomes $\left\{t_{1}, t_{2}, . . t_{y}\right\}$ defined from an existing crisp tree where $\boldsymbol{y}$ is the total number of outcomes. Fuzzy inference of $\boldsymbol{S}$ will involve an inference mechanism, $\boldsymbol{I} \boldsymbol{M}$ which consists of

an intersection function $f \wedge$, which takes in $\boldsymbol{V}$ and a $\mathrm{t}-$ norm TMin, which produces a set of minimum

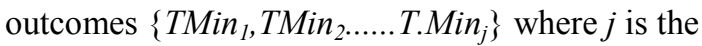
number of leaf nodes, and a union function $f \vee$, which combines output from $f \wedge$ to produce a maximum membership grade $\boldsymbol{O}$. Let $f \wedge, f \vee \boldsymbol{O} \in\{0.0,1\}$ consisting of real numbers, $\mathfrak{R}$.

i. Applying the fuzzy intersection function, $f \wedge$ This involves combining membership grades down all paths, from root to leaves within the tree.

$$
f \wedge\left(\left\{\mu_{1}, \mu_{2}, \ldots \mu_{v}\right\}\right) \rightarrow \text { TMin }\left\{\operatorname{TMin}_{1}, \text { TMin }_{2} \ldots . . T . \operatorname{Min}_{j}\right\}
$$

ii. Apply possibilistic strength of leaf node

The possibilistic strength of a leaf node represents the possibility that an example reaching a leaf node will have the same outcome as the leaf. Let $\boldsymbol{P}$ be a set of leaf possibilities $\left\{p_{1}, p_{2} \ldots p_{y}\right\}$ where $\boldsymbol{y}$ is the total number of leaf outcomes then

$$
\begin{gathered}
f \wedge\left(\left\{\mu_{1}, \mu_{2}, \ldots \mu_{v}\right\}\right) \rightarrow \text { TMin } \\
\left\{\left(\text { TMin }_{1} * p_{1}\right),\left(\text { TMin }_{2} * p_{2}\right) \ldots \ldots\left(\text { TMin }_{j} * p_{y}\right)\right\}
\end{gathered}
$$

Each leaf possibility $p_{y}$ is applied to the corresponding membership grade at each leaf node $\operatorname{TMin}_{j}$, after the intersection operation has been applied to combine grades of membership down one tree path.

iii. Applying fuzzy union function $f \vee$

The fuzzy union operator is applied in-order to combine the membership grades from all leaf nodes in order to produce a representative final grade of membership.

$$
f \vee\left(\left\{\left(\operatorname{TMin}_{1} * p_{1}\right),\left(\operatorname{TMin}_{2} * p_{2}\right) \ldots \ldots\left(\operatorname{TMin}_{j} * p_{y}\right) \rightarrow \boldsymbol{O}\right.\right.
$$

$\boldsymbol{O}$ is the fuzzy singleton used to determine the success of correct classification having taken place for $\boldsymbol{S}$.

4. Interpret and implement the tree as a set of Fuzzy IFTHEN rules. Each rule within the classifier may be defined as:

$$
\begin{aligned}
& R j_{1 . . j_{n}}: I F x_{1} \text { is } A_{1 j 1} \ldots . \text { AND } X_{n} \text { is } A_{n j n} \\
& \text { THEN } y \text { is } b_{j 1 . j n}, \\
& \text { Where } j 1=1,2, \ldots K_{1} ; \quad j n=1,2, \ldots, K n
\end{aligned}
$$

$R j_{1 . . j_{n}}$ is the label of each fuzzy if-then rule,

$b_{j 1 . j n}$, is the consequent real number and

$K$ is the number of fuzzy sub-spaces.

5. Optimize membership function and inference weightings (if applicable)

A full description of the original FIA can be found in [24].

\section{B. Defuzzification}

The unique characteristics of a specific classification problem will often determine the defuzzification strategy used. The experiments in this paper have been undertaken using discrete outcome data set. In this instance the fuzzy singleton is first used to construct the consequence part of the rule after the min intersection operator has been applied. Each consequence is then combined using the max fuzzy union operator to produce a real valued outcome.

\section{GENERATING A FUZZY DECISION TREE FROM CONVERSATIONAL TUTORIALS}

\section{A. Data Set}

75 undergraduate university students who had previous experience of SQL completed the formal ILS questionnaire and the Oscar CITS SQL Revision tutorial. During the tutorial their behavior and test scores were logged. Information relevant to the prediction of learning styles was captured in 41 different variables during the tutorial. The value of each variable in table II was determined by a series of logic rules (see Section II, B) being fired during the tutoring conversation. Table II shows all 41 variables within the data set that were captured. Each variable is associated with predicting at least one learning style dimension. For example, one variable, no interactions, captured the number of conversational interactions the user had with Oscar during the session. The variables Q5_choice and Q5steps 2 captured the student's approach to writing queries (i.e. whether they try all at once or want to try one step at a time). These two variables along with $Q^{9}$ choice and $Q^{9}$ steps 2 are indicative of the SEQUENTIAL/GLOBAL and SENSORY/INTUTIVE learning dimensions. Most learning 
style dimensions had an approximately even split of students, apart from the Visual/Verbal dimension where most students were Visual. This was not unexpected and in accordance with the ILS model [5] for students studying science and engineering based subjects.

TABLE II. OSCAR DATA SET DESCRIPTION

\begin{tabular}{|c|c|c|c|c|}
\hline Variable name & Type & $\begin{array}{c}\text { Values / } \\
\text { Range }\end{array}$ & Mean & $\begin{array}{c}\text { Standard } \\
\text { Deviation }\end{array}$ \\
\hline "Rules_VIS & Numeric & 1 to 11 & 6.733 & 2.767 \\
\hline Rules_VRB & Numeric & 0 to 11 & 3.400 & 2.427 \\
\hline Rules $\overline{3}$ _VIS & Numeric & 0 to 16 & 4.413 & 2.881 \\
\hline Rules3_VRB & Numeric & 0 to 12 & 3.053 & 2.117 \\
\hline Rules_s̄ EQ & Numeric & 1 to 11 & 6.200 & 2.307 \\
\hline Rules_GLO & Numeric & 1 to 10 & 4.933 & 1.848 \\
\hline Rules $\overline{3}$ SEQ & Numeric & 0 to 10 & 6.067 & 2.662 \\
\hline Rules3_GLO & Numeric & 0 to 8 & 4.347 & 1.573 \\
\hline Q5_choice & Discrete & 4 & & \\
\hline Q5_steps2 & Discrete & 3 & & \\
\hline Q9_choice & Discrete & 4 & & \\
\hline Q9_steps2 & Discrete & 3 & & \\
\hline Q4_detail & Discrete & 4 & & \\
\hline Q_prac & Numeric & 0.2 to 1 & 0.673 & 0.174 \\
\hline Q thep & Numeric & 0.125 to 1 & 0.678 & 0.183 \\
\hline Keywd_eg & Numeric & 0 to 3 & 0.04 & 0.346 \\
\hline Keywd_show & Numeric & 0 to 3 & 0.627 & 0.749 \\
\hline Keywd_see & Numeric & 0 to 1 & 0.013 & 0.115 \\
\hline Keywd_tell & Numeric & 0 to 2 & 0.04 & 0.257 \\
\hline Keywd_picture & Numeric & 0 to 1 & 0.013 & 0.115 \\
\hline Keywd_discuss & Numeric & 0 to 2 & 0.027 & 0.231 \\
\hline Rules_ĀCT & Numeric & 0 to 11 & 5.52 & 2.549 \\
\hline Rules_REF & Numeric & 0 to 11 & 3.013 & 2.883 \\
\hline Rules $\overline{3}$ ACT & Numeric & 0 to 10 & 3.907 & 1.62 \\
\hline Rules3 REF & Numeric & 0 to 3 & 3.267 & 0.6 \\
\hline Rules_S̄NS & Numeric & 0 to 11 & 4.72 & 3.451 \\
\hline Rules_INT & Numeric & 0 to 11 & 4.52 & 2.811 \\
\hline Rules $\overline{3}$ SNS & Numeric & 0 to 4 & 1.307 & 0.822 \\
\hline Rules3_INT & Numeric & 1 to 14 & 4.293 & 2.358 \\
\hline MCQ theory & Numeric & 0 to 1 & 0.524 & 0.37 \\
\hline MCQ pract & Numeric & 0 to 1 & 0.532 & 0.373 \\
\hline Duration & Numeric & $\begin{array}{l}845 \text { to } \\
14527\end{array}$ & 444.68 & 1637.56 \\
\hline Mean_Duration & Discrete & 2 & & \\
\hline Time_per_int & Numeric & 18 to 415 & 52.906 & 45.906 \\
\hline Mean_time_int & Discrete & 2 & & \\
\hline Read_time & Numeric & $\begin{array}{l}4.305 \text { to } \\
102.303\end{array}$ & 12.33 & 11.295 \\
\hline MeanRdTime & Discrete & 2 & & \\
\hline Wordcount & Numeric & 67 to 352 & 164.022 & 68.67 \\
\hline Mean_WC & Discrete & 2 & & \\
\hline No_interactions & Numeric & 24 to 84 & 48.84 & 9.895 \\
\hline Mean_interact & Discrete & 2 & & \\
\hline Class & Discrete & 2 & & \\
\hline
\end{tabular}

\section{B. Experimental Methodology}

For each experiment set, stratified 10-fold cross validation was used. For the purpose of this work, experiments were undertaken on two of the learning style dimensions: perception (sensory-intuitive) and understanding (sequentialglobal). Following the FIA methodology, first crisp decision trees were created from the data set using the statistical chisquare pruning technique with significance levels of $0.1 \%$ in order to produced highly optimized crisp trees which would allow good generalization to take place. Figure 3 shows a sample crisp decision tree for the sequential-global learning dimension. Table III, provides a high level description of variables used within the tree in Figure 3.

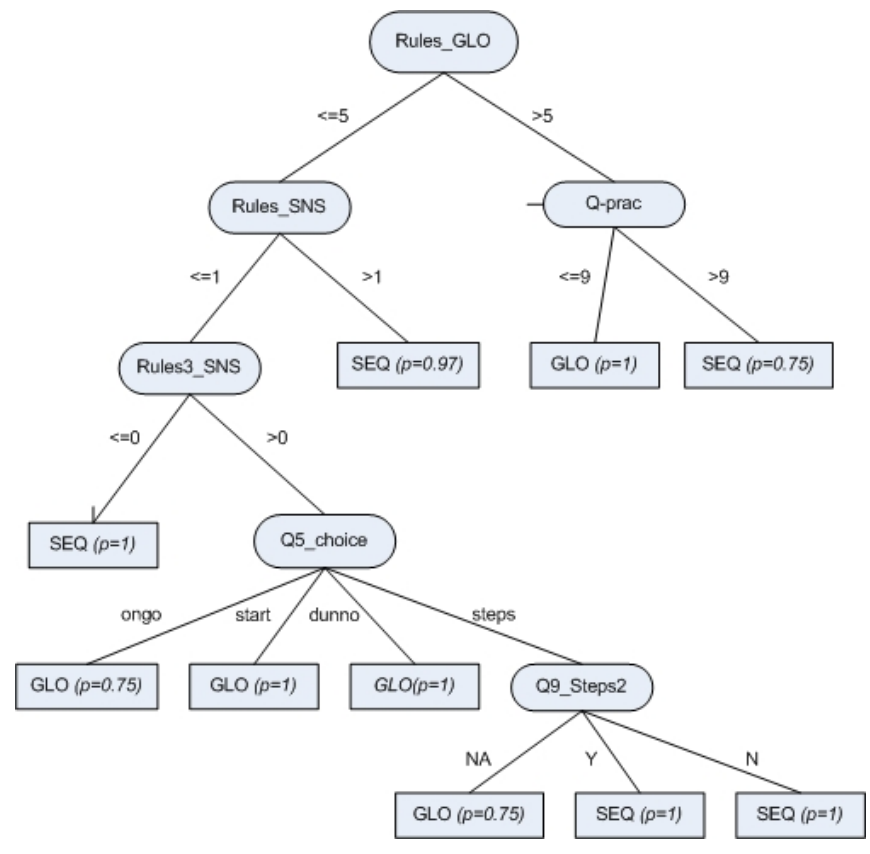

Fig. 3. Crisp tree representing sequential-global dimension

TABLE III. VARIABLE DESCRIPTION

\begin{tabular}{ll}
\hline Variable & Description \\
\hline Rules_GLO & $\begin{array}{l}\text { The number of user interactions which } \\
\text { mapped to Global logic rules that fired } \\
\text { during the tutoring conversation. }\end{array}$
\end{tabular}

Rules_SNS The number of user interactions which mapped to Sensory logic rules that fired during the tutoring conversation.

Rules3_SNS The number of Sensory rules that fired during user interaction AND whether the student got the answer right directly afterwards.

Q5_choice Records the approach taken by the student in the first attempt in answering a complex query (Q5).

Q9_Steps2 Records a change in approach taken by the student during their answer to complex query Q9.

$Q \_$prac Records the number of correct practical based questions during the tutorial.

Fuzzy membership functions were generated for both discrete and continuous attributes. Initial membership functions were selected to be equivalent to crisp sets with the initial fuzzy classifier system becoming an alternative representation of the decision tree. For experiments reported in this paper the amount of fuzzification either side of each tree node was increased linearly. Figure 4 shows an example 
membership function generated from the training data for the attribute RulesSen.

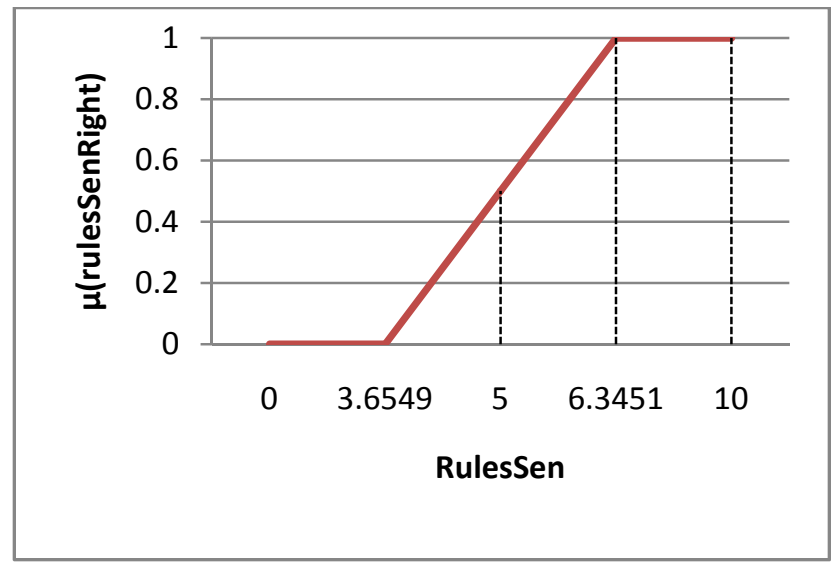

Fig. 4. $\mu$ (rulesSenRight)

Using a one-to-one mapping, each tree was then transformed into a set of Fuzzy IF-THEN rules. For example, the tree in figure 3 would be transformed into 10 rules. Zadeh's MinMax inference technique [33] was to combine grades of memberships of all rules within the fuzzy classification trees. In each experiment, results were generated using 10fold cross validation. The classification accuracy of the fuzzy classification tree can be defined as

$F_{1}=\frac{\sum_{i=1}^{\mathrm{i}=\mathrm{n}} \frac{\text { Numberof CorrectlyClassifiedRecordsin Class } i}{\text { TotalNumberof Recordsin Class } i}}{n}$

where $i=$ Class $\mathrm{i}$, and $n=$ Number of classes.

Accuracy was measured by comparing the system predicted learning style to the ILS questionnaire result of the same participant.

\section{RESULTS AND DISCUSSION}

Tables IV and V present the results of the sequential-global and sensory-intuitive dimensions respectively using three methods. In each table the average results of 10 fold cross validation are shown. For the sequential-global dimension (Table IV), columns \%AVG SEQ and \%AVG GLO show the \%accuracy of predicting sequential learners and global learners respectively, with the overall prediction accuracy for the sequential-global learning dimension being shown in the \%AVG SEQ/GLO column. Table V presents the results for the sensory-intuitive dimension in a similar way. In tables IV and V; OneVar gives the classification accuracy of using one variable predictor to classify the learning style; DT(C4.5) provides the classification accuracy of a crisp decision tree model where multiple variables are combined based upon their effect on the outcome; Fuzzy DT show the results obtained by the fuzzy classification trees generated by FIA.

\begin{tabular}{lccc}
\multicolumn{4}{c}{ TABLE IV SEQUENTIAL - GlOBAL } \\
\hline Method & \%AVG & $\%$ AVG & $\%$ AVG \\
& SEQ & GLO & SEQ/GLO \\
\hline OneVar (Q5choice) & 70 & 59 & 65 \\
DT (C4.5) & 80 & 70 & 76 \\
Fuzzy DT & 93 & 83 & 88 \\
\hline \multicolumn{4}{c}{} \\
& TABLE V SENSORY-INTUITIVE \\
\hline Method & SENG & $\%$ AVG & $\%$ AVG \\
& 70 & INT & SEN/INT \\
\hline OneVar(Q5choice) & 84 & 80 & 65 \\
DT (C4.5) & 98 & 73 & 82 \\
Fuzzy DT & & 59 & 86 \\
\hline
\end{tabular}

The results in tables IV and V show that in the case of both learning dimensions, when only one variable is used (OneVar); the same variable (Q5choice) was identified as being the most significant in terms of prediction accuracy. An initial observation of the results shows that correlation does exist between the two learning dimensions as sequential people like to learn step by step and sensory people like to go slowly and carefully, whereas global people like to jump straight in and intuitive people are fast and careless. The results in Tables IV for OneVar show that $70 \%$ of students who choose to answer the Question 9 query in the tutorial, one step at a time are Sequential learners. Using this variable (Q5choice) the Oscar CITS would predict that students in this group are Sequential learners and be accurate $70 \%$ of the time.

The results of inducing fuzzy classification trees yielded some interesting results with regards to the models that were produced. In modeling the sequential-global dimension, the attribute Rules_GLO (representing the number of global related rules that had fired during the conversation) was seen to be the most significant detector of learning style. However, what was surprising was the number of sensory attributes (Rules_SNS and Rules3_SNS) that were also included in the model. This again supported the correlation between sequential-global and sensory-intuitive dimensions.

The results in Tables IV and $\mathrm{V}$ also show that combining the attributes from the dataset generates a model which can be used to improve the prediction accuracy of the learner through the conversational agent tutorial. For the sequentialglobal dimension, the fuzzy classification trees produced gave a $23 \%$ improvement over prediction using one variable and a $12 \%$ increase over the crisp C4.5 decision tree. For the sensory-intuitive dimension, a $21 \%$ improvement was obtained over the one variable predictor and a $4 \%$ improvement over the crisp C4.5 decision tree. 


\section{CONCLUSION}

This paper proposes a new method which uses fuzzy classification trees to build a fuzzy predictive model in order to predict a students learning style through a conversational intelligent tutoring system. Using a dataset of 41 independent variables captured during the tutorial, experiments were carried out on two of the learning style dimensions: perception (sensory-intuitive) and understanding (sequential-global). The results have shown that the fuzzy predictive model has substantially increased the predictive accuracy of the Oscar CITS compared with the one variable predictor currently used within the system. Further work will incorporate the fuzzy predictive model into the adaptive Oscar CITS in order to provide an improved and more personalized learning experience.

\section{REFERENCES}

[1] Brusilovsky, P., Peylo, C.: Adaptive and Intelligent Web-based Educational Systems. Int.J. Artificial Intelligence in Education 13, 2003, pp.156-169.

[2] Ammar, M.B., Neji, M., Alimi, A.M., Gouarderes, G.: The Affective Tutoring System. Expert Systems with Applications 37, 2010, pp.3013-3023.

[3] Leontidis, M., Halatsis, C.: Integrating Learning Styles and Personality Traits into an Affective Model to Support Learner's Learning. In: Spaniol, M., et al. (eds.) Advances in Web Based Learning - ICWL 2009. LNCS, vol. 5686, 2009, pp. 225-34.

[4] Papanikolaou, K.A., Grigoriadou, M., Kornilakis, H., Magoulas, G.D.: Personalizing the Interaction in a Web-based Educational Hypermedia System: the case of INSPIRE. User Modeling and User-Adapted Interaction 13, 2003, pp.213-267.

[5] Felder, R., Silverman, L.K.: Learning and Teaching Styles in Engineering Education. J. Engineering Education 78(7), 1988, pp.674-681.

[6] Wang, H.C., Li, T.Y., Chang, C.Y.: A web-based tutoring system with styles-matching strategy for spatial geometric transformation. Interacting with Computers 18, 2006, pp.331-355.

[7] Cha, H.J., Kim, Y.S., Park, S.H., Yoon, T.B., Jung, Y.M., Lee, J.H.: Learning styles diagnosis based on user interface behaviours for the customization of learning interfaces in an intelligent tutoring system. In: Ikeda, M., Ashley, K.D., Chan, T.-W. (eds.) LNCS, vol. 4053, 2006, pp. 513-524.

[8] Spallek, H.: Adaptive Hypermedia: A New Paradigm for Educational Software. Advances in Dental Research 17(1), 2003, pp.38-42.

[9] Garcia, P., Amandi, A., Schiaffino, S., Campo, M.: Evaluating Bayesian networks' precision for detecting students' learning styles. Computers \& Education 49, 2007, pp.794-808.

[10] Kelly, D., Tangney, B.: Adapting to intelligence profile in an adaptive educational system. Interacting with Computers 18, 2006, pp.385409.

[11] Popescu, E.: An Artificial Intelligence Course Used to Investigate Students' Learning Style. In: Li, F., Zhao, J., Shih, T.K., Lau, R., Li, Q., McLeod, D. (eds.) LNCS, vol. 5145, 2008, pp. 122-131.

[12] Latham, A. Crockett, K. McLean, D, Edmonds, B. "Predicting Learning Styles in a Conversational Intelligent Tutoring System", in X. Luo et al (eds.), LNCS, vol. 6483, 2010, pp. 131-140.

[13] Crockett, K. Bandar, Z. O'Shea, J. Mclean, D. Goal Orientated Conversational Agents - the rocky road to commercialization. IEEE World Congress on Computational Intelligence, July 2010, pp.18201827.

[14] Crockett, K, O'Shea, J, Bandar, Z. Goal Orientated Conversational Agents: Applications to Benefit Society. Springer-Verlag, KES 2011, LNCS, 2011, in press.

[15] Weber, G., Brusilovsky, P.: ELM-ART: An adaptive versatile system for Web-based instruction Int. J. Artificial Intelligence in Education vol. 12(4), 2001, pp.351-384.
[16] Graesser, A., Chipman, P., Haynes, B.C., Olney, A.: AutoTutor: An Intelligent Tutoring System With Mixed-Initiative Dialogue. IEEE Trans. Education vol. 48(4), 2005, pp.612-618.

[17] Latham, A. Crockett, K, Mclean, D, Edmonds, B. Oscar: An Intelligent Adaptive Conversational Agent Tutoring System'. Springer-Verlag, KES 2011, LNCS, 2011, in press.

[18] Hawkes, L. Derry, S. Advances in local student modeling using informal fuzzy reasoning. International Journal of Human-Computer Studies, vol. 45(6), 1996, pp. 697-722.

[19] Xu, D., Wang, H., \& Su., K. (2002). Intelligent student profiling with fuzzy models. In HICSS'02, 35th Annual Hawaii international conference on system sciences, vol. 3, 2002.

[20] Garcia-Valdez, M. Alanis, A. Parra, B. Fuzzy inference for Learning Object Recommendation, IEEE International Conference on Fuzzy Systems, 2010, pp.1-6.

[21] Cabada, R.Z.; Estrada, M.L.B.; Garcia, C.A.R.; A Fuzzy-Neural Network for Classifying Learning Styles in a Web 2.0 and Mobile Learning Environment, 2009 Latin American Web Congress (la-web 2009), 2009, pp.177-182.

[22] Fazlollahtabar H.,Mahdavi, I. User/tutor optimal learning path in elearning using comprehensive neuro-fuzzy approach, Educational Research Review, vol.4(2), 2009, pp.142-155.

[23] Crockett, K. Bandar, Z., O’Shea, J, Fowdar, J. A fuzzy numeric inference strategy for classification and regression problems. KES Journal vol. 12(4), 2008, pp.255-269.

[24] Crockett, K., Bandar, Z., O’Shea, J. Mclean, D. On Constructing a Fuzzy Inference Framework using Crisp Decision Trees. Fuzzy Sets and Systems, vol. 157(21), 2006, pp.2809-2832.

[25] Marsala, C. Bouchon-Meunier, B. Ranking Attributes to Build Fuzzy Decision Trees: a Comparative Study of Measures. 2006 IEEE International Conference on Fuzzy Systems, 2006, pp.8436-8442.

[26] Chen, Yi-lai, Wang, T, Wang, B, Li, Z, A survey of fuzzy decision tree classifier, Fuzzy Information and Engineering, Springer Berlin Heidelberg, in co-publication with Fuzzy Information and Engineering Branch of the Operations Research Society of China, vol. 1(2), 2009, pp. $149-159$.

[27] Piero P. Bonissone, José Manuel Cadenas, M. Carmen Garrido, R. Andrés Díaz-Valladares: A fuzzy random forest. Int. J. Approx. Reasoning vol. 51(7), 2010, pp.729-747.

[28] Crockett, K. Bandar, Z. O'Shea, J. Fuzzification of Discrete Attributes From Financial Data in Fuzzy Classification Trees, IEEE International conference on Fuzzy Systems, South Korea, 2009, pp.1320-1325.

[29] Mitrovic, A.: An Intelligent SQL Tutor on the Web. Int. J. Artificial Intelligence in Education vol. 13, 2003, pp171-195.

[30] Melis, E., Andrès, E., Büdenbender, J., Frishauf, A., Goguadse, G., Libbrecht, P., Pollet, M., Ullrich, C.: ActiveMath: A web-based learning environment. Int. J. Artificial Intelligence in Education vol. 12(4), 2001, pp.385-407.

[31] Latham, A. Crockett, K. Mclean, D, Edmonds. B. Oscar: An Intelligent Conversational Agent Tutor to Estimate Learning Styles. IEEE World Congress on Computational Intelligence, July 2010, pp.2533-2540.

[32] Quinlan, J, R. C4.5: Programs for Machine Learning. Morgan Kaufmann Publishers, 1993.

[33] Zadeh, L. Knowledge Representation In Fuzzy Logic. In An Introduction To Fuzzy Logic Applications In Intelligent systems, eds. Yager, R and Zadeh, L, Kluwer Academic Publishers, 1992. 